\title{
Accurate Calculation of Magnetic Fields in the End Regions of Superconducting Accelerator Magnets Using the BEM-FEM Coupling Method
}

\author{
S. Kurz*, S. Russenschuck \\ *ITE, University of Stuttgart, Germany \\ CERN, Geneva, Switzerland
}

\begin{abstract}
In this paper a new technique for the accurate calculation of magnetic fields in the end regions of superconducting accelerator magnets is presented. This method couples Boundary Elements (BEM) which discretize the surface of the iron yoke and Finite Elements (FEM) for the modelling of the nonlinear interior of the yoke. The BEM-FEM method is therefore specially suited for the calculation of 3dimensional effects in the magnets, as the coils and the air regions do not have to be represented in the finite-element mesh and discretization errors only influence the calculation of the magnetization (reduced field) of the yoke. The method has been recently implemented into the CERNROXIE program package for the design and optimization of the LHC magnets. The field shape and multipole errors in the two-in-one LHC dipoles with its coil ends sticking out of the common iron yoke is presented.
\end{abstract}

\section{INTRODUCTION}

The design and optimization of the LHC magnets is dominated by the requirement of an extremely uniform field which is mainly defined by the layout of the superconducting coils. Even very small geometrical effects such as the insufficient keystoning of the cable, the insulation, grading of the current density in the cable due to different cable compaction and coil deformations due to collaring, cool down and electromagnetic forces have to be considered for the field calculation. In particular for the 3D case, commercial software has proven to be hardly appropriate for the field optimization of the LHC magnets. Therefore the ROXIE program package was developed at CERN for the design and optimization of the LHC superconducting magnets. Using the BEM-FEM coupling method [1] yields the reduced field in the aperture due to the magnetization of the iron yoke and avoids the representation of the coil in the FE-meshes.

\section{THE BEM-FEM COUPLING METHOD}

The total magnetic induction $\vec{B}$ in a certain point $\vec{\xi}$ in the aperture of the magnet can be decomposed into a contribution $\vec{B}_{\mathrm{S}}$ due to the superconducting coil and a contribution $\vec{B}_{\mathrm{R}}$ due to the magnetic yoke. If the fields are expressed in terms of the magnetic vector potential $\vec{B}=\operatorname{curl} \vec{A}$, then the decomposition into source and reduced contributions gives

$$
\vec{A}=\vec{A}_{\mathrm{S}}+\vec{A}_{\mathrm{R}}
$$

This approach has the following intrinsic advantages: (1) The coil field can be taken into account in terms of its source vector potential $\overrightarrow{A_{\mathrm{S}}}$, which can be obtained easily from the filamentary currents $I_{\mathrm{S}}$ by means of Biot-Savart type integrals without meshing of the coil. (2) The BEMFEM coupling method allows for the direct computation of the reduced vector potential $\vec{A}_{\mathrm{R}}$ rather than the total vector potential $\vec{A}$. Then numerical errors do not influence the dominating contribution $\vec{A}_{\mathrm{S}}$ due to the superconducting coil. (3) The surrounding air region needs not to be meshed at all. This simplifies the preprocessing and avoids artificial boundary conditions at some "far" boundaries. Moreover, the geometry of the permeable parts can be modified without taking care of the mesh in the surrounding air region. This strongly supports the feature based, parametric geometry modelling which is required for mathematical optimization.

When the BEM-FEM coupling method is applied, only the magnetic sub-domain $\Omega_{i}$ which coincides with the magnetic yoke has to be discretized by finite elements. Iron saturation effects can then be dealt with within the finite element framework. The nonmagnetic sub-domain $\Omega_{a}$ which represents the surrounding air region and the excitation coil is treated by the boundary element method. Only the common boundary $\Gamma$ needs to be discretized by boundary elements. The source vector potential $\vec{A}_{\mathrm{S}}$ can be obtained from the filamentary current $I_{\mathrm{S}}$ by means of the Biot-Savart type integral

$$
\vec{A}_{\mathrm{S}}=\mu_{0} I_{\mathrm{S}} \oint_{\mathcal{C}} u^{*} \mathrm{~d} \vec{l} .
$$

In (2), the Green's function $u^{*}$ is the fundamental solution of the Laplace equation, which is in $2 \mathrm{D}$

$$
u^{*}=-\frac{1}{2 \pi} \ln |\vec{x}-\vec{\xi}|
$$

and in 3D

$$
u^{*}=\frac{1}{4 \pi|\vec{x}-\vec{\xi}|} .
$$

$\vec{\xi}$ is the evaluation point of $\overrightarrow{A_{\mathrm{S}}}$ and $\vec{x}$ is the integration point on $\mathcal{C}$.

\subsection{The FEM part}

Inside the magnetic domain $\Omega_{\mathrm{i}}$ a gauged vector-potential formulation is applied. With Maxwell's equations 
$\operatorname{curl} \vec{H}=\vec{J}$ and $\operatorname{div} \vec{B}=0$ for magnetostatic problems, the constitutive equation

$$
\vec{B}=\mu(\vec{H}) \cdot \vec{H}=\mu_{0}(\vec{H}+\vec{M})
$$

and the vector-potential formulation $\vec{B}=\operatorname{curl} \vec{A}$ we get

$$
\frac{1}{\mu_{0}} \operatorname{curl} \operatorname{curl} \vec{A}=\vec{J}+\operatorname{curl} \vec{M}
$$

Introducing the penalty term $-\operatorname{grad} \frac{1}{\mu_{0}} \operatorname{div} \vec{A}$ the weak formulation reads

$$
\begin{array}{r}
\frac{1}{\mu_{0}} \int_{\Omega_{\mathrm{i}}}(\operatorname{curl} \vec{w} \cdot \operatorname{curl} \vec{A}+\operatorname{div} \vec{w} \operatorname{div} \vec{A}) \mathrm{d} \Omega_{\mathrm{i}} \\
-\oint_{\Gamma_{\text {ai }}} \vec{w} \cdot\left(\frac{1}{\mu 0} \operatorname{curl} \vec{A} \times \vec{n}-\frac{1}{\mu_{0}}(\operatorname{div} \vec{A}) \vec{n}\right) \mathrm{d} \Gamma_{\text {ai }} \\
-\int_{\Omega_{\mathrm{i}}} \vec{M} \cdot \operatorname{curl} \vec{w} \mathrm{~d} \Omega_{\mathrm{i}}=\int_{\Omega_{\mathrm{i}}} \vec{w} \cdot \vec{J} \mathrm{~d} \Omega_{\mathrm{i}}
\end{array}
$$

which can be transformed to

$$
\begin{array}{r}
\frac{1}{\mu_{0}} \int_{\Omega_{\mathrm{i}}} \operatorname{grad}\left(\vec{A} \cdot \vec{e}_{a}\right) \cdot \operatorname{grad} w_{a} \mathrm{~d} \Omega_{\mathrm{i}} \\
-\frac{1}{\mu_{0}} \oint_{\Gamma_{\mathrm{ai}}}\left(\frac{\partial \vec{A}}{\partial n_{\mathrm{i}}}-\left(\mu_{0} \vec{M} \times \vec{n}_{\mathrm{i}}\right)\right) \cdot \vec{w}_{a} \mathrm{~d} \Gamma_{\mathrm{ai}}= \\
\int_{\Omega_{\mathrm{i}}} \vec{M} \cdot \operatorname{curl} \vec{w}_{a} \mathrm{~d} \Omega_{\mathrm{i}}+\int_{\Omega_{\mathrm{i}}} \vec{w}_{a} \cdot \vec{J} \mathrm{~d} \Omega_{\mathrm{i}}
\end{array}
$$

$a=1,2,3$ and

$$
\vec{w}_{1}=\left(\begin{array}{c}
w_{1} \\
0 \\
0
\end{array}\right), \vec{w}_{2}=\left(\begin{array}{c}
0 \\
w_{2} \\
0
\end{array}\right), \vec{w}_{3}=\left(\begin{array}{c}
0 \\
0 \\
w_{3}
\end{array}\right) .
$$

The continuity condition of $\vec{H}_{\mathrm{t}}$ on the boundary between iron and air leads to

$$
\frac{\partial \vec{A}^{\mathrm{FEM}}}{\partial n_{\mathrm{i}}}-\left(\mu_{0} \vec{M} \times \vec{n}_{\mathrm{i}}\right)+\frac{\partial \vec{A}^{\mathrm{BEM}}}{\partial n_{\mathrm{a}}}=0 .
$$

$\vec{n}_{\mathrm{i}}$ is the normal vector on $\Gamma_{\mathrm{ai}}$ pointing out of the FEM domain $\Omega_{\mathrm{i}}$ and $\vec{n}_{\mathrm{a}}$ is the normal vector on $\Gamma_{\text {ai }}$ pointing out of the BEM domain $\Omega_{\mathrm{a}}$. The boundary integral term on the boundary between iron and air $\Gamma_{\mathrm{ai}}$ in (7) serves as the coupling term between the BEM and the FEM domain. Let us now assume, that the normal derivative on $\Gamma_{\mathrm{ai}}$

$$
\vec{Q}_{\Gamma_{\mathrm{ai}}}=-\frac{\partial \vec{A}_{\Gamma_{\mathrm{ai}}}^{\mathrm{BEM}}}{\partial n_{\mathrm{a}}}
$$

is given a priori. If the domain $\Omega_{\mathrm{i}}$ is discretized into finite elements $\left(C^{0}\right.$-continuous, isoparametric 20 -noded hexahedron elements are used), and the Galerkin method is applied to the weak formulation, then a non linear system of equations is obtained

$$
\left(\begin{array}{ccc}
{\left[K_{\Omega_{\mathrm{i}} \Omega_{\mathrm{i}}}\right]} & {\left[K_{\Omega_{\mathrm{i}} \Gamma_{\mathrm{ai}}}\right]} & 0 \\
{\left[K_{\Gamma_{\mathrm{ai}} \Omega_{\mathrm{i}}}\right]} & {\left[K_{\Gamma_{\mathrm{ai}} \Gamma_{\text {ai }}}\right]} & {[T]}
\end{array}\right)\left(\begin{array}{l}
\left\{\vec{A}_{\Omega_{\mathrm{i}}}\right\} \\
\left\{\vec{A}_{\Gamma_{\mathrm{ai}}}\right\} \\
\left\{\vec{Q}_{\Gamma_{\text {ai }}}\right\}
\end{array}\right)=\left(\begin{array}{l}
0 \\
0
\end{array}\right)
$$

with all nodal values of $\vec{A}_{\Omega_{\mathrm{i}}}, \vec{A}_{\Gamma_{\mathrm{ai}}}$ and $\vec{Q}_{\Gamma_{\mathrm{ai}}}$ grouped in arrays. The subscripts $\Gamma_{\mathrm{ai}}$ and $\Omega_{\mathrm{i}}$ refer to nodes on the boundary and in the interior of the domain, respectively. The domain and boundary integrals in the weak formulation yield the stiffness matrices $[K]$ and the boundary matrix $[T]$. The stiffness matrices depend on the local permeability distribution in the nonlinear material. All the matrices in (12) are sparse.

\subsection{The BEM part}

By definition the BEM domain $\Omega_{\mathrm{a}}$ does not contain any iron and therefore $\vec{M}=0$ and $\mu=\mu_{0}$. One more integration by parts of the weak integral form (8) and choosing the Cartesian components of the vector weighting function as the fundamental solution of the Laplace equation yields

$$
\begin{aligned}
\frac{\Theta}{4 \pi} \vec{A}+\oint_{\Gamma_{\text {ai }}} \vec{Q}_{\Gamma_{\text {ai }}} u^{*} \mathrm{~d} \Gamma_{\text {ai }} & +\oint_{\Gamma_{\text {ai }}} \vec{A}_{\Gamma_{\text {ai }}} q^{*} \mathrm{~d} \Gamma_{\text {ai }} \\
& =\int_{\Omega_{\mathrm{a}}} \mu_{0} \vec{J} u^{*} \mathrm{~d} \Omega_{\mathrm{a}}
\end{aligned}
$$

with the abbreviation $q^{*}=\frac{\partial u^{*}}{\partial n_{a}}$. The right hand side of Eq. (13) is a Biot-Savart type integral for the source vector potential $\overrightarrow{A_{\mathrm{s}}}$. The vector potential $\vec{A}$ at any arbitrary point $\vec{r}_{0} \in \Omega_{\mathrm{a}}$ can be computed from (13) once the vector potential $\vec{A}_{\Gamma_{\mathrm{ai}}}$ and its normal derivative $\vec{Q}_{\Gamma_{\mathrm{ai}}}$ on the boundary $\Gamma_{\mathrm{ai}}$ are known. $\Theta$ is the solid angle enclosed by the domain $\Omega_{\mathrm{a}}$ in the vicinity of $\vec{r}_{0}$. For the discretization of the boundary $\Gamma_{\mathrm{ai}}$ into individual boundary elements $\Gamma_{\mathrm{ai}, j}, C^{0}$-continuous, isoparametric 8 -noded quadrilateral boundary elements are used. The functions $\vec{A}_{\Gamma_{\mathrm{ai}}}$ and $\vec{Q}_{\Gamma_{\mathrm{ai}}}$ are expanded with respect to the element shape functions and (13) can be rewritten in terms of the nodal data of the discrete model,

$$
\frac{\Theta}{4 \pi} \vec{A}=\vec{A}_{\mathrm{s}}-\left\{\vec{Q}_{\Gamma_{\mathrm{ai}}}\right\} \cdot\{g\}-\left\{\vec{A}_{\Gamma_{\mathrm{ai}}}\right\} \cdot\{h\} .
$$

In (14), $g$ results from the boundary integral with the kernel $u^{*}$ and $h$ results from the boundary integral with the kernel $q^{*}$. The discrete analogue of the Fredholm integral equation can be obtained from (14) by successively putting the evaluation point $\overrightarrow{r_{0}}$ at the location of each nodal point $\overrightarrow{j_{j}}$. This procedure is called point-wise collocation and yields a linear system of equations,

$$
[G]\left\{\vec{Q}_{\Gamma_{\text {ai }}}\right\}+[H]\left\{\vec{A}_{\Gamma_{\text {ai }}}\right\}=\left\{\vec{A}_{\mathrm{s}}\right\} .
$$

In (15), $\left\{\overrightarrow{A_{\mathrm{s}}}\right\}$ contains the values of the source vector potential at the nodal points $\vec{r}_{j}, j=1,2, \ldots$ The matrices $[G]$ and $[H]$ are unsymmetric and fully populated.

\subsection{The BEM-FEM Coupling}

An overall numerical description of the field problem can be obtained by complementing the FEM description (12) 
by the BEM description (15) resulting in

$$
\left(\begin{array}{ccc}
{\left[K_{\Omega_{\mathrm{i}} \Omega_{\mathrm{i}}}\right]} & {\left[K_{\Omega_{\mathrm{i}} \Gamma_{\mathrm{ai}}}\right]} & 0 \\
{\left[K_{\Gamma_{\mathrm{ai}} \Omega_{\mathrm{i}}}\right]} & {\left[K_{\Gamma_{\mathrm{ai}} \Gamma_{\mathrm{ai}}}\right]} & {[T]} \\
0 & {[H]} & {[G]}
\end{array}\right)\left(\begin{array}{c}
\left\{\vec{A}_{\Omega_{\mathrm{i}}}\right\} \\
\left\{\vec{A}_{\Gamma_{\mathrm{ai}}}\right\} \\
\left\{\vec{Q}_{\Gamma_{\mathrm{ai}}}\right\}
\end{array}\right)=\left(\begin{array}{c}
0 \\
0 \\
\left\{\vec{A}_{\mathrm{s}}\right\}
\end{array}\right) .
$$

Equation (15) gives exactly the missing relationship between the Dirichlet data $\left\{\vec{A}_{\Gamma_{\mathrm{ai}}}\right\}$ and the Neumann data $\left\{\vec{Q}_{\Gamma_{\mathrm{ai}}}\right\}$ on the boundary $\Gamma_{\mathrm{ai}}$. It can be shown [2] that this procedure yields the correct physical interface conditions, the continuity of $\vec{n} \cdot \vec{B}$ and $\vec{n} \times \vec{H}$ across $\Gamma_{\text {ai }}$.

\section{RESULTS}

The Large Hadron Collider (LHC) to be built at CERN requires high-field superconducting magnets to guide the counter-rotating beams in the LEP tunnel with a circumference of about $27 \mathrm{~km}$. The design and optimization of these magnets is dominated by the requirement of an extremely uniform field, which is mainly defined by the layout of the superconducting coils. In order to study, with a fast turnaround rate, the influence of individual coil parameters, the pre-stress in the coil, the collar material and the yoke structures, a short-model dipole program was established at CERN. 20 single-aperture models and 3 double-aperture models have been built and tested since mid 1995 in addition to 5 long-model prototypes built in European industry. Unlike in the main dipole prototypes with a magnetic length of $14.2 \mathrm{~m}$, the field quality in the center of the short dipole models (with a coil length of $1.05 \mathrm{~m}$ and the length of the magnetic yoke of only $402 \mathrm{~mm}$ ) is affected by the coil ends. In order to study systematic effects in the field quality due to manufacturing tolerances, and coil deformations due to assembly and cool-down of the magnet it is necessary to calculate, with a high precision, the $3 \mathrm{D}$ integrated multipole field errors. This is important as the measurement coil used at present has a length of about $200 \mathrm{~mm}$ (half the length of the magnetic yoke). Fig. 1 shows the geometric model of the coiltest facility (CTF). Fig. 2 shows the relative multipole components $b_{3}, b_{5}$ and $b_{7}$ (related to the main field $B_{1}$ of $8.2452 \mathrm{~T}$ calculated at $11530 \mathrm{~A}$ for the two dimensional model, at $17 \mathrm{~mm}$ ref. radius, in units of $10^{-4}$ ) as a function of the $\mathrm{z}$-position. $\mathrm{z}=0$ is the center of the magnet. The iron yoke ends at $\mathrm{z}=201 \mathrm{~mm}$.

At about nominal current, the $b_{3}$ component in the center of the short model dipole does not reach the $2 \mathrm{D}$ cross-section value of 4.2 units due to globally different saturation effects. At $4000 \mathrm{~A}$ the $b_{3}$ shows a local enhancement of only about 0.3 units near the aperture and reaches its crosssection value of 2.9 units about $100 \mathrm{~mm}$ inwards the yoke. The $b_{5}$ and in particular $b_{7}$ and higher order multipoles are little influenced by three-dimensional effects.

\section{CONCLUSION}

The BEM-FEM method is specially suited for the calculation of $3 \mathrm{D}$ effects in superconducting magnets, as the coils

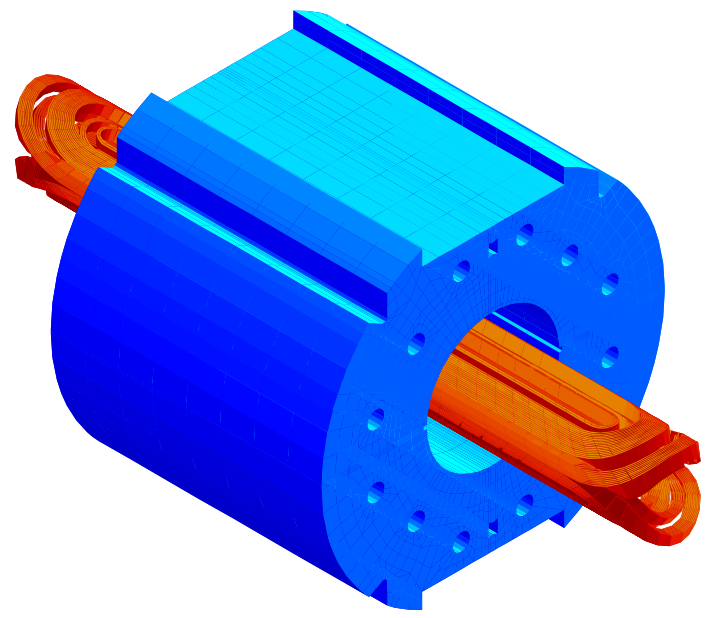

Figure 1: ROXIE model of the CTF

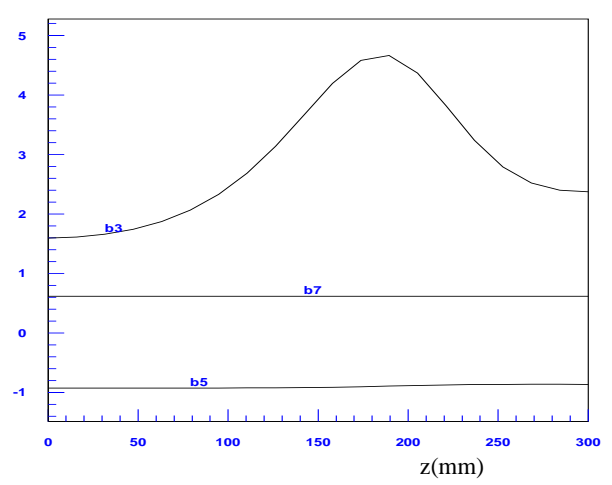

Figure 2: Relative multipole components (at $\mathrm{r}=17 \mathrm{~mm}$, in units of $10^{-4}$ ) as a function of the axial position

and the air regions do not have to be represented in the finite-element mesh and discretization errors only influence the calculation of the yoke magnetization. The method has been applied to the calculation of multipole errors in the short dipole models for the LHC. Results show that the models are representative for the long dipole prototypes only at low and medium excitation. At nominal excitation, the sextupole measured in the center of the magnet is more than one unit lower than in the center of the long magnets.

\section{REFERENCES}

[1] J. Fetzer, S. Abele, and G. Lehner. Die Kopplung der Randelementmethode und der Methode der finiten Elemente zur Lösung dreidimensionaler elektromagnetischer Feldprobleme auf unendlichem Grundgebiet. Archiv für Elektrotechnik, 76(5):361-368, 1993.

[2] S. Kurz, J. Fetzer, and W. Rucker. Coupled BEM-FEM methods for 3D field calculations with iron saturation. Proceedings of the First International ROXIE users meeting and workshop, 1998. 